Journal for ImmunoTherapy of Cancer

\section{Histiocyte predominant myocarditis resulting from the addition of interferon gamma to cyclophosphamide-based lymphodepletion for adoptive cellular therapy}

To cite: Schroeder BA, Black RG, Spadinger S, et al. Histiocyte predominant myocarditis resulting from the addition of interferon gamma to cyclophosphamide-based lymphodepletion for adoptive cellular therapy. Journal for ImmunoTherapy of Cancer 2020;8:e00247. doi:10.1136/ jitc-2019-000247

Accepted 02 March 2020
Check for updates

(C) Author(s) (or their employer(s)) 2020. Re-use permitted under CC BY-NC. No commercial re-use. See rights and permissions. Published by BMJ.

For numbered affiliations see end of article.

Correspondence to Dr Seth M Pollack; spollack@fredhutch.org

\section{ABSTRACT}

Background Adoptive cellular therapy (ACT) is a promising treatment for synovial sarcoma (SS) with reported response rates of over $50 \%$. However, more work is needed to obtain deeper and more durable responses. SS has a 'cold' tumor immune microenvironment with low levels of major histocompatibility complex (MHC) expression and few T-cell infiltrates, which could represent a barrier toward successful treatment with ACT. We previously demonstrated that both MHC expression and T-cell infiltration can be increased using systemic interferon gamma (IFN- $\gamma$ ), which could improve the efficacy of ACT for SS.

Case presentation We launched a phase I trial incorporating four weekly doses of IFN- $\gamma$ in an ACT regimen of high-dose cyclophosphamide (HD Cy), NY-ESO-1-specific T cells, and postinfusion low-dose interleukin (IL)-2. Two patients were treated. While one patient had significant tumor regression and resultant clinical benefit, the other patient suffered a fatal histiocytic myocarditis. Therefore, this cohort was terminated for safety concerns.

Conclusion We describe a new and serious toxicity of immunotherapy from IFN- $\gamma$ combined with HD Cy-based lymphodepletion and low-dose IL-2. While IFN- $\gamma$ should not be used concurrently with HD Cy or with low dose IL-2, IFN- $\gamma$ may still be important in sensitizing SS for ACT. Future studies should avoid using IFN- $\gamma$ during the immediate period before/after cell infusion.

Trial registration numbers NCT04177021, NCT01957709, and NCT03063632.

\section{BACKGROUND}

Synovial sarcoma (SS) is a soft-tissue malignancy with few available treatments, and overall survival is often less than 2 years when metastatic. ${ }^{2}$ However, SS maybe an ideal tumor type for adoptive cellular therapy (ACT), given frequent and homogeneous expression of NY-ESO-1. Importantly, the NY-ESO-1 protein is routinely expressed only in testicular germ cells and in some cancers, but not in healthy adult tissues. ${ }^{3} 4$ Multiple trials targeting the A*0201 restricted NY-ESO-1 (157-165) epitope have demonstrated greater than $50 \%$ responses, with some showing durable and/or reinducible responses at the time of progression. ${ }^{5-7}$ Unfortunately, in almost all cases, patients who initially respond ultimately progress. We previously reported that SS has an immunologically 'cold' tumor microenvironment, with few infiltrating $\mathrm{T}$ cells, and low levels of gene expression related to antigen presentation, including constituents of the major histocompatibility complex (MHC). ${ }^{8}$ However, treatment of patients with SS with systemic interferon gamma (IFN- $\gamma$ ) increased MHC expression and increased T-cell infiltration in matched tumor biopsies in a phase 0 clinical trial. ${ }^{9}$

Here we describe a pilot study integrating weekly IFN- $\gamma$ with ACT in the context of high-dose cyclophosphamide (HD Cy)based lymphodepletion and low-dose IL-2. While the first patient (IFN\#1) had an impressive regression of pulmonary tumors, the second patient (IFN\#2) developed a fatal histiocytic myocarditis. Although we concluded that this conditioning regimen was not safe moving forward, IFN- $\gamma$ could still play an important role in future immunotherapy trials; thus, future investigators must be aware of this toxicity.

\section{METHODS}

Patients, leukapheresis acquisition and clinical T-cell products

HLA typing of patient samples was performed at the Puget Sound Blood Center (PSBC). The interventional and pilot studies using 


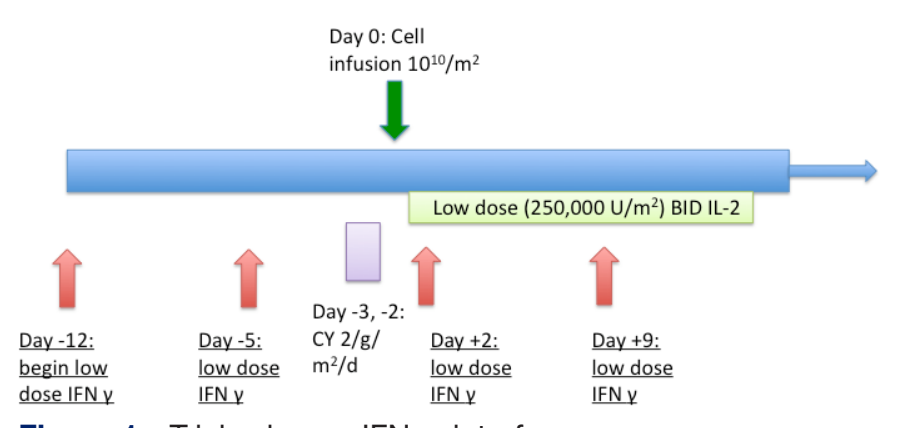

Figure 1 Trial schema. IFN- $\gamma$, interferon gamma.

either NY-ESO-1-specific T cells or IFN- $\gamma$ were registered with clinicaltrials.gov.

Leukapheresis was performed at either PSBC or at the University of Washington (UW) General Clinical Research Center if the patient expressed HLA A*0201, had an Eastern Cooperative Oncology Group (ECOG) performance status of 0 or 1 , and if tumor biopsies stained positive for NY-ESO- 1 in $>25 \%$ of cells by immunohistochemistry (IHC). Subsequent processing of leukapheresed product was performed at FHCRC, including the cell processing facility (CPF), under current good manufacturing practice (GMP) guidelines. This form of ACT using peripheral blood as a source of antigen-specific $\mathrm{T}$ cells is known as endogenous T-cell (ETC) therapy and yields a T-cell product of near uniform specificity with central memory properties. ${ }^{10}$ ETC has been used in first-in-human studies targeting tumor-associated antigens with evidence of clinical efficacy. ${ }^{1112}$

To facilitate detailed functional analysis, an aliquot of T-cell product was removed from the CPF and then expanded in the research laboratory. In vitro assays were used to assess recognition of target cells pulsed with various peptide concentrations and to assess cytokine production secondary to antigen stimulation. Cell surface phenotype and $\mathrm{T}$ cell receptor beta chain variable region (TCR V $\beta$ ) sequencing were performed on aliquots from the second expansion. ${ }^{13}$ Specificity of expanded T-cell products was confirmed using chromium release assays performed on day 12 of the rapid expansion protocol. T2 target cells were cultured alone for 2 hours or in media with target peptide; when relevant, the HLA A*0201+, NY-ESO-1+tumorline, Mel A375 (A375), was also used as target cell.

\section{Flow cytometry and functional analysis of antigen specific $\mathrm{T}$ cells}

Cell surface phenotype of persisting cells was determined by costaining with NY-ESO-1 tetramer (FHCRC, Seattle, Washington, USA) and fluorochrome-conjugated monoclonal antibodies (mAbs). Multicolor flow cytometry data were collected using a LSRII (Becton Dickinson, Franklin Lakes, New Jersey, USA) and FlowJo software. For staining, cells were fixed and stained for CD8 (BD Bioscience, Franklin Lakes, New Jersey, USA).

\section{TCR V $\beta$ sequencing}

TCR V $\beta$ sequencing and normalization was performed on suspensions of tumor-infiltrating lymphocytes, as well as paraffin-mbedded specimens by Adaptive Biotechnologies (Seattle, Washington, USA). The number of total cells, T cells, T-cell fraction, the number of unique rearrangements, and clonality were calculated for each sample as previously described. ${ }^{14}$

\section{Immunohistochemistry}

Immunohistochemical analyses of myocardium for CD3, CD4, CD8, CD20, and CD68 expression were performed using an automated immunostainer (Bond III) and the following mAbs: CD3 (clone LN10, dilution 1:100; Novacastra, Newcastle, UK), CD4 (clone SP35, dilution 1:25; Cell Marque, Rocklin, California, USA), CD8 (clone 4B11, dilution 1:200; Novacastra), CD20 (clone L26, dilution 1:800; Dako, Glostrup, Denmark), and CD68 (clone Kp-1, dilution 1:1000; Cell Marque). Tonsils were used as positive controls.

Immunohistochemical expression to confirm eligibility of patients for treatment with NY-ESO-1-specific T cells was performed at the UW Pathology Lab with mAb specific for NY-ESO-1 (1:100, E978 clone; Life Technologies, Grand Island, New York, USA), CD3 (clone LN10, dilution 1:200; Novacastra), and MHC class I (1:8000, EMR8-5 clone; Abcam, Cambridge, Massachusetts, USA).

\section{RESULTS}

To test whether systemic weekly IFN- $\gamma$ could be safely combined with ACT using NY-ESO-1-specific T cells, we designed a phase I trial using weekly IFN- $\gamma\left(100 \mu \mathrm{g} / \mathrm{m}^{2}\right)$ beginning 2 weeks prior to cell infusion and continuing for 2 weeks after the cell infusion. Lymphodepletion consisted of HD Cy $\left(2 \mathrm{~g} / \mathrm{m}^{2} /\right.$ day $)$ on days -3 and -2 , prior to the $\mathrm{T}$ cell infusion (day 0 ). To augment in vivo proliferation, low-dose IL-2 $\left(250000 \mathrm{U} / \mathrm{m}^{2}\right.$ every 12 hours $)$ was administered for 2 weeks after the infusion (figure 1).

\section{Patient 1}

IFN\#1 was a man in his mid-40s with intractable pain in his left shoulder. He visited a local emergency department, and an investigative chest X-ray showed a hilar mass. Subsequent CT of the chest, abdomen, and pelvis again demonstrated the hilar mass, along with a $2.5 \times 3.6 \mathrm{~cm}$ pelvic mass. Biopsy showed diffuse expression of Bcl-2, vimentin, CD99, and neoplastic cells expressing TLE-1, indicative of SS. He received two cycles of doxorubicin and ifosfamide, with disease progression. Next, he received two cycles of trabectedin, again with disease progression (table 1).

Multiple brain metastases were identified, and the patient underwent gamma knife radiation. Given that his tumor demonstrated strong homogenous NY-ESO-1 expression and that he was HLA-A0201+, he was enrolled in the ACT trial. 


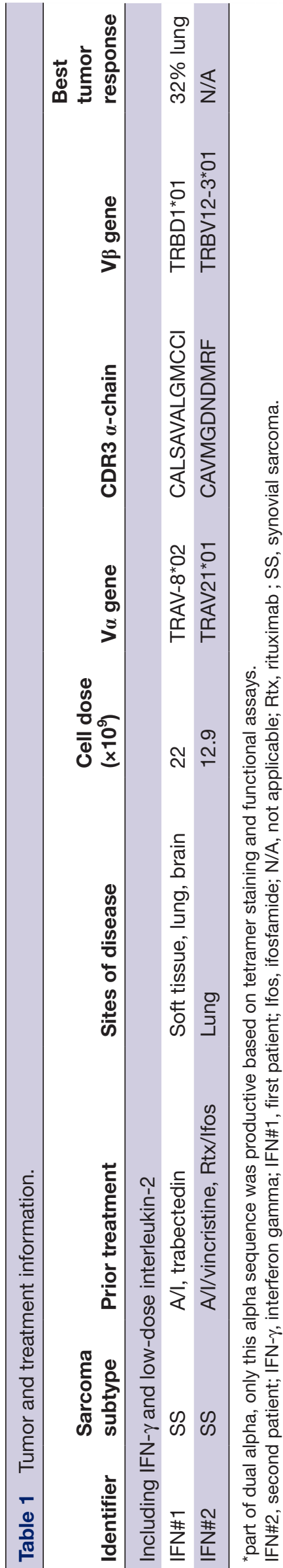

Peristence of Tetramer Positive Cells
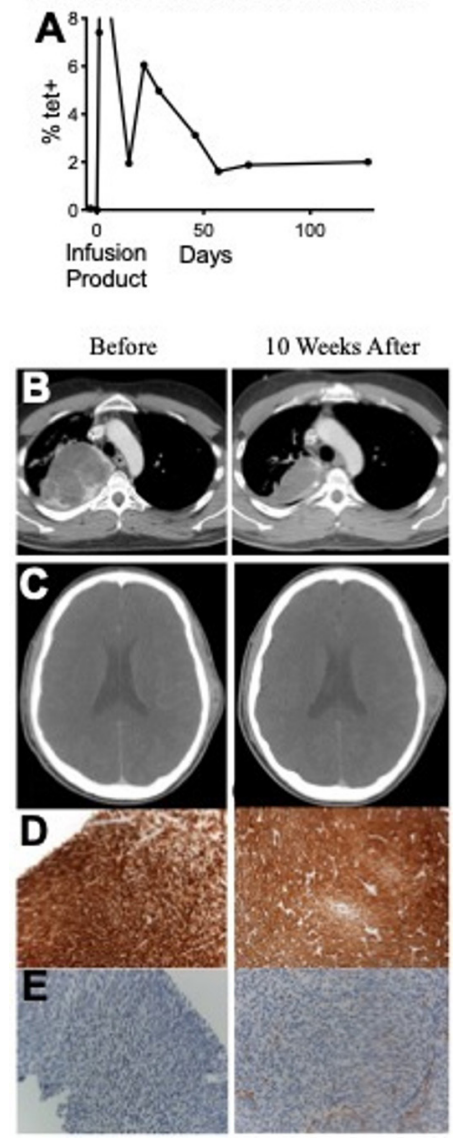

Figure 2 (A) Persistence of transferred T cells as absolute values. Percent tetramer positive derived from peripheral CD8+ T cells. (B) CT scan showing pretreatment and post-treatment lung. (C) Forehead soft tissue mass. (D) Pretreatment lung (left) and post-treatment forehead mass (right) stained for NY-ESO-1. (E) Lung (left) stained for HLA$A B C$, forehead mass (right) stained for HLA-ABC.

Despite the aggressive metastatic chemoresistant disease, 4 weeks after T-cell infusion, all six lung tumors showed a measurable decrease in size. A hilar lymph node decreased from $3.0 \times 3.0 \mathrm{~cm}$ to $1.9 \times 3.0 \mathrm{~cm}$, and the small pleural-based lung nodules also decreased in size. The most dramatic response was in a large right upper lobe (RUL) tumor that previously received $3600 \mathrm{cGy}$ of radiation 3 months prior to ACT. This mass was $10.8 \times 8.1 \mathrm{~cm}$ prior to radiation and remained stable at $10.4 \times 8.0 \mathrm{~cm}$ 3 months after radiation therapy. However, 4 weeks after T-cell therapy, the tumor decreased markedly in size to $6.9 \times 5.3 \mathrm{~cm}$, and by 10 weeks to $6.9 \times 4.5 \mathrm{~cm}$ (figure $2 \mathrm{~B}$ ). Importantly, biopsy of lung lesion pretreatment and forehead lesion post-treatment were stained for NY-ESO-1 and MHC class I. The post-treatment sample showed both increased NY-ESO-1 staining (figure 2D) and increased MHC class I expression (figure 2E). No lung tumors (including subcentimeter nodules) had progressed 4 weeks after T-cell therapy. Subjectively, he reported a marked improvement in fatigue and respiratory status following ACT and even returned to work after missing weeks because of fatigue. He experienced common 
self-limited toxicities of HD Cy (leukopenia, anemia, malaise, and mild mucositis), IFN- $\gamma$ and IL-2 (fatigue, malaise, and myalgias), but no unexpected toxicities.

Prior to T-cell infusion, NY-ESO-1 tet $^{+} \mathrm{T}$ cells were undetectable in the blood $(<0.01 \%$ of CD $8+$ cells $)$. Four weeks after infusion, following complete reconstitution of the absolute lymphocyte and white blood cell counts and cyclophosphamide conditioning, NY-ESO-1 tet ${ }^{+}$ $\mathrm{T}$ cells represented $5 \%$ of all peripheral CD8+ $\mathrm{T}$ cells (figure 2A). At 10 weeks postinfusion, this cell population remained detectable.

Despite the marked response of the pulmonary metastases, the overall response was mixed as a soft tissue lesion on his forehead progressed 10 weeks after ACT (figure 2C). To investigate the lack of response at some tumor sites, his forehead lesion was excised. The pathology demonstrated homogenous staining of NY-ESO-1 by IHC, but few infiltrating T cells were found and the tissue lacked MHC class I expression (data not shown), which was thought to be responsible for the lack of response and may indicate that the impact of IFN- $\gamma$ in the tumor microenvironment is not durable.

\section{Patient 2}

The IFN\#2 was a man in his mid-40s who first noticed lower back pain. His pain progressed, and a small lump eventually developed. MRI at an outside hospital showed a right flank mass measuring $7.8 \times 8.7 \times 12.9 \mathrm{~cm}$. Subsequent biopsy demonstrated poorly differentiated SS. He underwent six cycles of neoadjuvant doxorubicin with ifosfamide and vincristine, followed by surgical resection. $\mathrm{He}$ also received radiation and concurrent ifosfamide for two cycles after the surgical resection. Unfortunately, 2 years later during routine surveillance, lung metastases were discovered in both lungs. After discussing multiple treatment options, the patient elected for ACT.

Before trial enrollment, his poorly controlled type 2 diabetes was noted with a hemoglobin Alc of $10.1 \%$, complicated by diabetic nephropathy and a baseline serum creatinine of $1.4 \mathrm{mg} / \mathrm{dL}(1.5 \mathrm{mg} / \mathrm{dL}$ was the trial max). Prior to ACT, a cardiac stress echocardiogram was performed and deemed normal. His ECOG performance status was 1.

On trial initiation, he experienced no symptoms from the single-agent IFN- $\gamma$ and tolerated Cy well. He had no immediate complications during T-cell infusion. Two days following T-cell infusion, he reported fatigue and was found to have an elevated creatinine of 1.9, attributed to $\mathrm{Cy}$ and an underlying renal dysfunction. He received intravenous hydration with normal saline in the outpatient infusion center, and his creatinine returned to baseline. On the evening between days 3 and 4 post-T cell infusion, he developed malaise, a cough with pink-tinged sputum, and a fever to $101^{\circ} \mathrm{F}$. CBC performed earlier that day confirmed an appropriate neutrophil count. He took acetaminophen at home and elected not to go to the emergency room, with planned follow-up at the clinic in 1-2 days. On day 4 after T-cell infusion, he suddenly lost consciousness and was found in cardiac arrest. Resuscitation was attempted but was unsuccessful.

Autopsy revealed disease that was more extensive than originally appreciated on CT with innumerable tiny $(<0.5 \mathrm{~cm})$ bilateral lung metastases. Autopsy also showed a myocarditis with an immune infiltrate primarily composed of CD68+ cells (figure 3D) and CD3+ cells (figure 3B). Almost no CD4+ or CD8+ cells were detected (data not shown). Since the infusion product was greater than $98 \% \mathrm{CD} 8+$, his myocarditis was attributed to the conditioning regimen, further complicated by his preexisting comorbidities. T-cell receptor (TCR) sequencing from myocardial tissue was attempted, but an insufficient number of reads were observed. This was consistent with the IHC that showed only a small fraction of infiltrating $\mathrm{T}$ cells, again suggesting that $\mathrm{T}$ cell-induced myocardial toxicity was not the cause of death. We also confirmed that the heart tissue did not express NY-ESO-1 and concluded that the addition of IFN- $\gamma$ to HD Cy and low-dose IL-2 as administered in this regimen was not safe, and this cohort was terminated.

\section{DISCUSSION AND CONCLUSIONS}

A variety of treatment modalities including immune checkpoint blockade, tumor-associated macrophage phenotype modulation, chimeric antigen and highaffinity T-cell receptors, along with vaccine therapies, are all under investigation for sarcomas. ${ }^{15}$ ACT targeting NY-ESO-1 is a promising treatment for SS. ${ }^{5-7}$ NY-ESO-1 has also been safely targeted using vaccines with potential clinical benefit in sarcoma. ${ }^{16}{ }^{17}$ However, SS has a cold tumor immune microenvironment illustrated by low levels of MHC expression and few T-cell infiltrates, which could impede meaningful responses. We previously showed that both MHC expression and T-cell infiltration can be increased using systemic IFN- $\gamma$ and thus could improve the efficacy of ACT for SS. ${ }^{18}$ IFN- $\gamma$ likely also increases programmed death-ligand 1 (PD-L1) expression in SS tumors, and attempts to combine IFN- $\gamma$ with programmed cell death protein 1 (PD-1) inhibition are ongoing. However, our results show that novel combinations of immunotherapeutic regimens must be explored cautiously, even when using treatments with well-established safety profiles.

In the original studies of Cy, cardiotoxicity was the doselimiting toxicity, but this is unusual with routine doses of $2 \mathrm{~g} / \mathrm{m}^{2} /$ day $\times 2$ days used in ACT trials. ${ }^{19}{ }^{20}$ Cy-related myocarditis is thought to arise from direct damage to the myocardium and vessels, resulting in edema, hemorrhage and the formation of microthrombi. ${ }^{21-23}$ Hemorrhage and microthrombi were not observed in this case. Instead, a profound infiltrate of CD68+ histiocytes was discovered. While IFN- $\gamma$ is Food and Drug Administration approved and has been used safely in the treatment of thousands of patients, IFN- $\gamma$ can activate macrophages and lead to cardiac trafficking in murine models. ${ }^{24}{ }^{25}$ It may be possible that a subclinical Cy-related myocarditis 


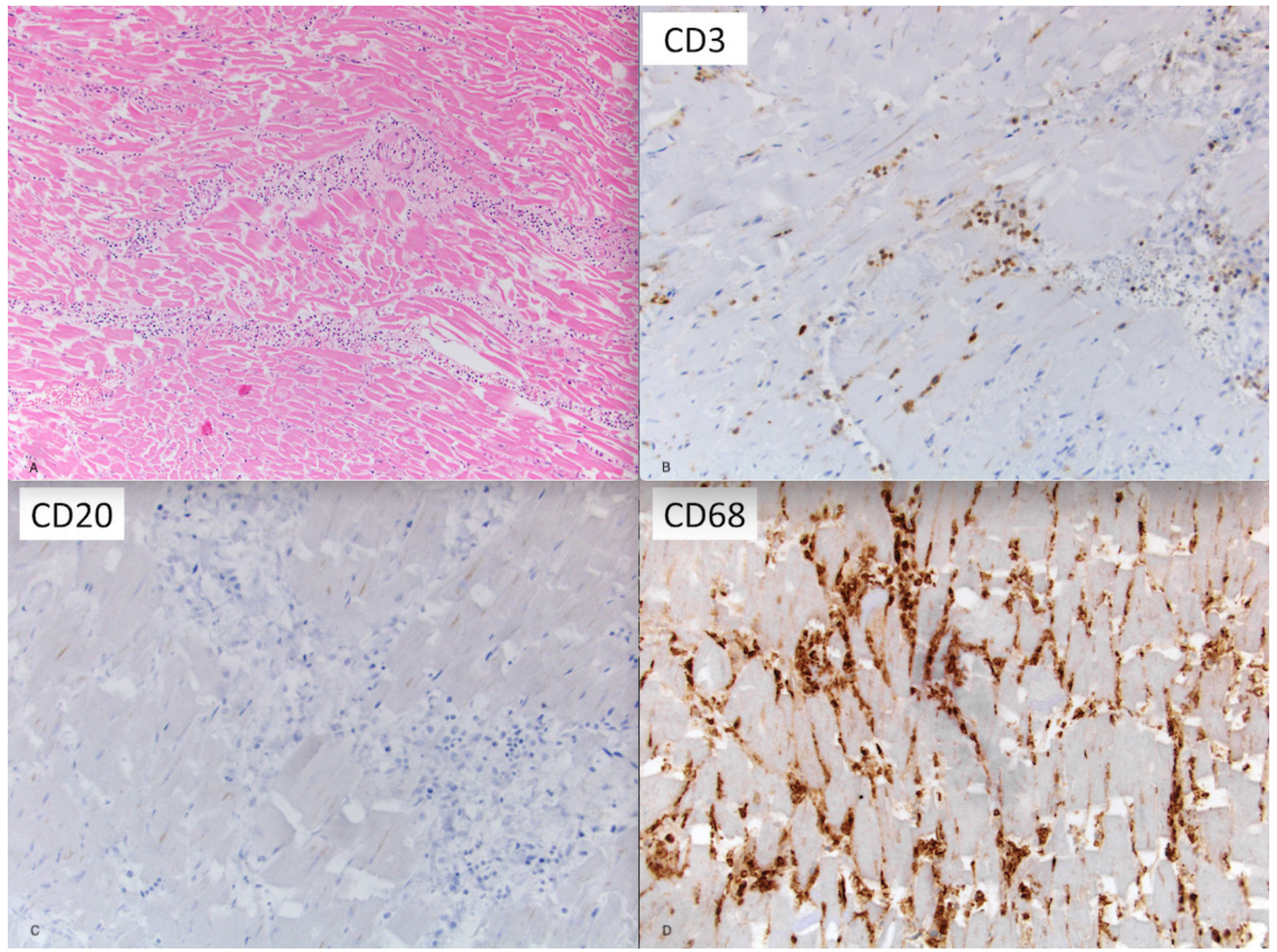

Figure 3 Histological sections of the heart at autopsy demonstrate myocyte necrosis and marked mononuclear inflammation (A, H\&E $\times 200$ ). Immunohistochemical stains highlight a mixed population of inflammatory cells composed of predominantly CD68+ histiocytes (D) and few CD3 +T cells (B). CD20 stain does not demonstrate a significant B-cell population (C).

was made clinically relevant through IFN- $\gamma$-induced macrophage stimulation.

Although we conclude that IFN- $\gamma$ should not be used concurrently with HD Cy or low-dose IL-2, IFN- $\gamma$ may still play an important role in sensitizing SS for ACT. Interestingly, another recent ACT study showed the combination of interferon alpha and tumor-infiltrating lymphocyte (TIL) therapy, including Cy pretreatment in a similar dose, reinfusion of TILs, and postinfusion of IL-2 in a higher dose, was found to be safe in 12 patients in a published article by Andersen $e t a l .{ }^{26}$ However, future studies should avoid using IFN- $\gamma$ during the immediate period around cell infusion and instead should consider alternative schedules, such as beginning IFN- $\gamma$ weeks after cell infusion or at the time of progression.

\section{Author affiliations}

${ }^{1}$ Clinical Research Division, Fred Hutchinson Cancer Research Center, Seattle, Washington, USA

${ }^{2}$ Virginia Mason Medical Center, Seattle, Washington, USA

${ }^{3}$ Fred Hutchinson Cancer Research Center, Seattle, Washington, USA

${ }^{4}$ Pathology, University of Washington Medical Center, Seattle, Washington, USA
${ }^{5}$ Orthopedic Surgery, University of Texas Health Science Center at Houston, Houston, Texas, USA

${ }^{6}$ Oncology, University of Washington Medical Center, Seattle, Washington, USA ${ }^{7}$ Sarcoma, Royal Marsden Hospital NHS Trust, London, UK

${ }^{8}$ Melanoma Medical Oncology, University of Texas MD Anderson Cancer Center, Houston, Texas, USA

Twitter Cassian Yee @tcellsrus and Seth M Pollack @immunosarc

Contributors BAS compiled and analyzed clinical data and helped prepare the manuscript. RGB, SS, SZ, KK, and JC performed experiments. JGM performed pathology. EUC, RLJ and SMP cared for the patient. SMP developed the novel trial. $C Y$ and SRR provided leadership and contributed to the preparation of the manuscript.

Funding This work was supported by the Gilman Sarcoma Foundation as well as K23CA175167.

Competing interests None declared.

Patient consent for publication Not required.

Ethics approval Patients were enrolled on protocols approved by the Fred Hutchinson Cancer Research Center Institutional Review Board for either clinical trials or tissue and blood procurement, and provided informed consent in accordance with the Declaration of Helsinki.

Provenance and peer review Not commissioned; externally peer reviewed. 
Open access This is an open access article distributed in accordance with the Creative Commons Attribution Non Commercial (CC BY-NC 4.0) license, which permits others to distribute, remix, adapt, build upon this work non-commercially, and license their derivative works on different terms, provided the original work is properly cited, appropriate credit is given, any changes made indicated, and the use is non-commercial. See http://creativecommons.org/licenses/by-nc/4.0/.

\section{ORCID iDs}

Brett A Schroeder http://orcid.org/0000-0001-6019-2022

Sydney Spadinger http://orcid.org/0000-0002-5160-9794

Seth M Pollack http://orcid.org/0000-0002-2466-0607

\section{REFERENCES}

1 Riedel RF, Jones RL, Italiano A, et al. Systemic anti-cancer therapy in synovial sarcoma: a systematic review. Cancers 2018;10. doi:10.3390/cancers10110417. [Epub ahead of print: 01 Nov 2018].

2 Moreau L-C, Turcotte R, Ferguson P, et al. Myxoid \round cell liposarcoma (MRCLS) revisited: an analysis of 418 primarily managed cases. Ann Surg Oncol 2012;19:1081-8.

3 Jungbluth AA, Antonescu CR, Busam KJ, et al. Monophasic and biphasic synovial sarcomas abundantly express cancer/testis antigen NY-ESO-1 but not MAGE-A1 or CT7. Int J Cancer 2001;94:252-6.

4 Pollack SM, Jungbluth AA, Hoch BL, et al. Ny-Eso-1 is a ubiquitous immunotherapeutic target antigen for patients with myxoid/round cell liposarcoma. Cancer 2012;118:4564-70.

5 Robbins PF, Morgan RA, Feldman SA, et al. Tumor regression in patients with metastatic synovial cell sarcoma and melanoma using genetically engineered lymphocytes reactive with NY-ESO-1. J Clin Oncol 2011;29:917-24.

6 Robbins PF, Kassim SH, Tran TLN, et al. A pilot trial using lymphocytes genetically engineered with an NY-ESO-1-reactive T-cell receptor: long-term follow-up and correlates with response. Clin Cancer Res 2015;21:1019-27.

7 D'Angelo SP, Melchiori L, Merchant MS, et al. Antitumor Activity Associated with Prolonged Persistence of Adoptively Transferred NY-ESO-1 ${ }^{\mathrm{c} 259} \mathrm{~T}$ Cells in Synovial Sarcoma. Cancer Discov 2018;8:944-57.

8 Pollack SM, He Q, Yearley JH, et al. T-Cell infiltration and clonality correlate with programmed cell death protein 1 and programmed death-ligand 1 expression in patients with soft tissue sarcomas. Cancer 2017;123:3291-304.

9 Zhang S, Kohli K, Black RG, et al. Systemic interferon- $\gamma$ increases $\mathrm{MHC}$ class I expression and T-cell infiltration in cold tumors: results of a phase 0 clinical trial. Cancer Immunol Res 2019;7:1237-43.

$10 \mathrm{Li} \mathrm{Y,} \mathrm{Bleakley} \mathrm{M,} \mathrm{Yee} \mathrm{C.} \mathrm{II-21} \mathrm{influences} \mathrm{the} \mathrm{frequency,} \mathrm{phenotype,}$ and affinity of the antigen-specific CD8 T cell response. J Immunol 2005;175:2261-9.
11 Chapuis AG, Roberts IM, Thompson JA, et al. T-Cell therapy using Interleukin-21-Primed cytotoxic T-cell lymphocytes combined with cytotoxic T-cell lymphocyte antigen-4 blockade results in longterm cell persistence and durable tumor regression. J Clin Oncol 2016;34:3787-95.

12 Yee C, Lizee G, Schueneman AJ. Endogenous T-cell therapy: clinical experience. Cancer J 2015;21:492-500.

13 Pollack SM, Jones RL, Farrar EA, et al. Tetramer guided, cell sorter assisted production of clinical grade autologous NY-ESO-1 specific CD8(+) T cells. J Immunother Cancer 2014;2:36.

14 Robins HS, Srivastava SK, Campregher PV, et al. Overlap and effective size of the human CD8+ T cell receptor repertoire. Sci Trans/ Med 2010; :;2:ra64. 2.

15 Pollack SM, Ingham M, Spraker MB, et al. Emerging targeted and immune-based therapies in sarcoma. J Clin Oncol 2018;36:125-35.

16 Pollack SM, Lu H, Gnjatic S, et al. First-In-Human treatment with a dendritic Cell-targeting lentiviral Vector-expressing NY-ESO-1, LV305, induces deep, durable response in refractory metastatic synovial sarcoma patient. J Immunother 2017;40:1-306.

17 Somaiah N, Block MS, Kim JW, et al. First-In-Class, first-in-human study evaluating LV305, a dendritic-cell tropic lentiviral vector, in sarcoma and other solid tumors expressing NY-ESO-1. Clin Cancer Res 2019;25:5808-17.

18 Zhang S, Kohli K, Black G, et al. Systemic interferon gamma increases $\mathrm{MCH}$ class one expression and T-cell infiltration in cold tumors: results of a phase 0 clinical trial. Cancer Immunol Res 2019;8:1237-43.

19 Goldberg MA, Antin JH, Guinan EC, et al. Cyclophosphamide cardiotoxicity: an analysis of dosing as a risk factor. Blood 1986;68:1114-8.

20 Higgins AY, O'Halloran TD, Chang JD. Chemotherapy-Induced cardiomyopathy. Heart Fail Rev 2015;20:721-30.

21 Appelbaum F, Strauchen JA, Graw RG, et al. Acute lethal carditis caused by high-dose combination chemotherapy. A unique clinical and pathological entity. Lancet 1976;1:58-62.

22 Katayama M, Imai Y, Hashimoto $\mathrm{H}$, et al. Fulminant fatal cardiotoxicity following cyclophosphamide therapy. J Cardiol 2009;54:330-4.

23 Shanholtz C. Acute life-threatening toxicity of cancer treatment. Crit Care Clin 2001;17:483-502

24 Reifenberg K, Lehr H-A, Torzewski M, et al. Interferon-Gamma induces chronic active myocarditis and cardiomyopathy in transgenic mice. Am J Pathol 2007;171:463-72.

25 Torzewski M, Wenzel P, Kleinert $\mathrm{H}$, et al. Chronic inflammatory cardiomyopathy of interferon $\gamma$-overexpressing transgenic mice is mediated by tumor necrosis factor- $\alpha$. Am J Pathol 2012;180:73-81.

26 Andersen R, Borch TH, Draghi A, et al. T cells isolated from patients with checkpoint inhibitor-resistant melanoma are functional and can mediate tumor regression. Ann Oncol 2018;29:1575-81. 